\title{
Early Diagnosis of Ocular Hypertension Using a Low-Intensity Laser Irradiation Test
}

\author{
Boris T. Ivandic, M.D., ${ }^{1,2}$ Nasreen N. Hoque, M.Sc., ${ }^{3}$ and Tomislav Ivandic, M.D. ${ }^{3}$
}

\begin{abstract}
Objective: We investigated the potential use of low-intensity laser irradiation (LILI) as a diagnostic tool for identifying hypertensive eyes at risk of glaucoma. Background data: The diagnosis of early-stage ocular hypertension is particularly difficult to establish. Methods: This study of a case series included 123 healthy subjects with normal vision. The intraocular pressure (IOP) was determined before (baseline) and $30 \mathrm{~min}$ after a 30-sec irradiation of the limbus area with laser light $(780 \mathrm{~nm} ; 7.5 \mathrm{~mW} ; 292 \mathrm{~Hz}$ modulation). Results: Baseline IOP was $>21 \mathrm{~mm} \mathrm{Hg}$ in 44 of 211 eyes (20.9\%), consistent with ocular hypertension. LILI decreased the mean IOP by $6.2 \mathrm{~mm} \mathrm{Hg}(-25.7 \% ; p<0.001$; paired $t$ test $)$ in these eyes. The remaining 167 eyes $(79.1 \%)$ exhibited a normotensive IOP $\leq 21 \mathrm{~mm} \mathrm{Hg}$. LILI reduced the mean IOP by $2.9 \mathrm{~mm} \mathrm{Hg}(-17.1 \%$; $p<0.001)$ in these eyes, but there were different response patterns: 1$)$ the IOP did not change $(27.0 \%) ; 2)$ the IOP was reduced by the same extent in both eyes $(32.3 \%)$; 3 ) initial IOP differences between left and right eyes became level and the absolute IOP was reduced to a lower level that was identical in both eyes (18.0\%); and 4) the initial difference in IOP between the left and right eye persisted despite LILI (22.7\%). Conclusion: LILI lowers IOP, even in normotensive eyes. This effect may be useful to determine the individual physiological IOP and to diagnose latent ocular hypertension in eyes with presumably normotensive IOP.
\end{abstract}

\section{Introduction}

G LAUCOMA, A CHRONIC, progressive eye disease, is found in about $2 \%$ of the population aged 40 or above. It is estimated that more than 70 million people are affected across the world. ${ }^{1}$ Although there are still different classifications, and even definitions, glaucoma is traditionally considered an optic nerve head disease associated with progressive loss of vision and increased intraocular pressure (IOP). ${ }^{1,2}$

The diagnosis of advanced glaucoma is usually straightforward; it is based on the characteristic pathological excavation of the optic nerve disc (glaucomatous excavation), typical defects of the visual field, and increased IOP. Functional and anatomical alterations are seen as direct consequences of the increased $\mathrm{IOP}^{3-6}$ although this association is controversial. $^{7-9}$ An increased IOP is the most important risk factor for damage to the optic nerve. The level of the IOP is, however, not always correlated with the clinical picture causing some diagnostic uncertainty. A "typical" glaucomatous visual field with a glaucomatous excavation and a "statistically normotensive" IOP $(\leq 21 \mathrm{~mm} \mathrm{Hg})$ may be found as well as the sole increase in IOP $>21 \mathrm{~mm} \mathrm{Hg}$ without these symptoms, which is termed ocular hypertension. Early diagnosis of glaucoma is even more difficult, in spite of advances in electrophysiology ${ }^{10}$ and perimetry, ${ }^{11-13}$ because the individual physiological IOP and its day-to-day variability, as well as other risk factors are unknown. ${ }^{14}$ Attempts to diagnose early-stage glaucoma by tonography or provocation tests, like water drinking and administration of caffeine or other drugs, have proved unreliable. ${ }^{15,16}$

Low-intensity laser irradiation (LILI), which, in contrast to surgical laser applications, does not cause any damage to tissues, ${ }^{17}$ has been widely used in experimental and small clinical studies. Recently, we reported that LILI improved vision in patients with both early and advanced stage, agerelated macular degeneration. ${ }^{18}$

In this study of a case series we examined the potential use of LILI as a tool to determine the individual physiological IOP. The aim was to differentiate between normoand hypertensive eyes by classifying changes in IOP after irradiation of the limbus area of the eye with a weak laser.

\footnotetext{
${ }^{1}$ University of Heidelberg, Otto-Meyerhof Centre, Heidelberg, Germany.

${ }^{2}$ DIAneering, Diagnostics Engineering and Research GmbH, Heidelberg, Germany.

${ }^{3}$ Medical Centre, Munich, Germany.
} 


\section{Materials and Methods}

\section{Study subjects}

This study of a case series was conducted in accordance with the Helsinki Declaration. Informed consent was obtained in writing from all subjects before enrollment. Data were collected at a single investigational site in Germany and analyzed retrospectively. Study subjects were included in the study if their eyes were apparently healthy (i.e., without obvious pathological findings) as determined by direct ophthalmoscopy and biomicroscopy, in particular without glaucomatous changes of the anterior or posterior eye segments. Subjects were excluded if they presented with eye or systemic disorders or if they were using medication for any disease.

Upon study entry, the baseline IOP was determined using applanation tonometry (Goldmann applanation tonometer; Zeiss, Jena, Germany). Tonometry was repeated $30 \mathrm{~min}$ after LILI.

\section{Laser treatment}

For LILI a semiconductor laser diode with continuous emission at $780 \mathrm{~nm}(7.5 \mathrm{~mW})$ was fitted with collimating optics (elliptic spot area $6.28 \mathrm{~mm}^{2}$ ) in a handheld casing. The laser diode was driven by an apparatus that provided lowfrequency switching of the laser beam $(292 \mathrm{~Hz}, 1: 1$ duty cycle) and automatic power control (Bimed, Munich, Germany). A safe minimal laser power for use in this study was previously determined by examination of enucleated pig eyes. $^{18}$

After determining the baseline IOP, each eye (random sequence) was irradiated for $30 \mathrm{sec}\left(0.22 \mathrm{~J} / \mathrm{cm}^{2}\right)$ in a circular fashion in the area of the limbus area at a distance of $1 \mathrm{~cm}$. Ten subjects (20 eyes) were randomly selected to receive mock treatment (control group): the laser beam was directed above the nose without contacting the eye, which was in adduction. An acoustical signal every $10 \mathrm{sec}$ was interpreted by the subjects as treatment. During the treatment period, no other therapy was applied. Safety and tolerability of the treatment were determined by interview, direct ophthalmoscopy, and biomicroscopy.

\section{Data analysis}

IOP before and $30 \mathrm{~min}$ after LILI were compared to calculate the absolute and relative changes in IOP. A paired $t$ test was performed to examine the statistical significance of changes in IOP.

\section{Results}

The physiological variability of the IOP during a 30-min period was first examined in a separate group of 30 subjects (12 men and 18 women with a mean age of $53.7 \pm 5.4$ years), who did not receive LILI treatment. This initial experiment confirmed that the IOP remained virtually unchanged: mean IOPs were $14.1 \pm 0.4 \mathrm{~mm} \mathrm{Hg}$ and $14.1 \pm 0.6 \mathrm{~mm} \mathrm{Hg}$ at baseline and at the second tonometry $30 \mathrm{~min}$ later, respectively.

\section{Variable changes in IOP after LILI}

In total, 123 healthy subjects (64 men, 59 women) with a mean age of $59.6 \pm 12.3$ years were included in the study. All subjects (109 right and 102 left eyes) were treated using LILI. Ten subjects ( 6 women, 4 men) with a mean age of $53.2 \pm 6.1$ years were mock treated and served as controls ( $n=20$ eyes).

Two separate groups of subjects were formed based on the baseline IOP, i.e., before treatment with LILI (Table 1).

At baseline, 44/211 eyes (22/123 subjects) had an IOP $>21 \mathrm{~mm} \mathrm{Hg}$ (mean IOP $24.1 \mathrm{~mm} \mathrm{Hg}$ ) consistent with definite ocular hypertension (group 1). The remaining 167/211 eyes (101/123 subjects) exhibited an IOP $\leq 21 \mathrm{~mm} \mathrm{Hg}$ (mean IOP $17.0 \mathrm{~mm} \mathrm{Hg}$ ), which was considered statistically "normotensive" (group 2). In this group the IOP was either identical in both eyes (100 of 167 eyes; 59.9\%) or different comparing left and right eye (67 of 167 eyes; $40.1 \%$ ).

Thirty minutes after LILI, the mean IOP decreased from 24.1 to $17.9 \mathrm{~mm} \mathrm{Hg}$ in hypertensive group 1; this decrease $(-25.7 \%)$ was statistically significant $(p<0.001$; pairwise comparison to baseline IOP values). In the "normotensive" group 2 the mean IOP decreased from 17.0 to $14.1 \mathrm{~mm} \mathrm{Hg}$ $30 \mathrm{~min}$ after LILI. Although smaller, this change $(-2.9 \mathrm{~mm}$ $\mathrm{Hg} ;-17.0 \%$ ) was still highly significant $(p<0.001$; pairwise comparison to baseline IOP values). A detailed analysis revealed different response patterns in group 2 (Table 1): in 100 of 167 eyes with identical IOP in both eyes, the mean IOP remained virtually unchanged in 45 eyes (mean IOP of $15.5 \mathrm{~mm} \mathrm{Hg}$ ) before and $30 \mathrm{~min}$ after LILI (group 2A), and

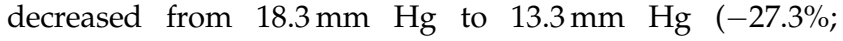
$p<0.001$ ) in 55 eyes (group 2B). IOP differed between left and right eyes in 67 eyes $30 \mathrm{~min}$ after LILI: 30 eyes exhibited a significant decrease of the mean IOP from 17.0 to $13.3 \mathrm{~mm}$

Table 1. Intraocular Pressure (IOP) 30 min After Low-Intensity Laser Irradiation (LILI) in Eyes Classified as Hypertensive (Group 1, $N=44$ ) and Normotensive (Group 2, $N=167$ ) at Baseline

\begin{tabular}{|c|c|c|c|c|c|}
\hline \multirow[b]{2}{*}{ IOP at baseline } & \multirow{2}{*}{$\frac{\text { Group } 1}{>21 m m ~ H g}$} & \multicolumn{4}{|c|}{ Group $2^{\mathrm{a}}$} \\
\hline & & \multicolumn{4}{|c|}{$\leq 21 \mathrm{~mm} \mathrm{Hg}$} \\
\hline Subgroup & 1 & $2 \mathrm{~A}$ & $2 \mathrm{~B}$ & $2 \mathrm{C}$ & $2 \mathrm{D}$ \\
\hline Eyes, $n$ & 44 & 45 & 55 & 30 & 37 \\
\hline IOP before LILI, $\mathrm{mm} \mathrm{Hg}$ & 24.1 & 15.5 & 18.3 & 17.0 & 17.2 \\
\hline IOP after LILI, $\mathrm{mm} \mathrm{Hg}$ & 17.9 & 15.5 & 13.3 & 13.3 & 14.3 \\
\hline Change in IOP, $\mathrm{mm} \mathrm{Hg}$ & -6.2 & 0.0 & -5.0 & -3.7 & -2.9 \\
\hline Change in IOP, $\%$ & -25.7 & 0.0 & -27.3 & -21.8 & -16.9 \\
\hline
\end{tabular}

${ }^{a}$ Please see text for an explanation of the subgroups. 
Table 2. Classification of Ocular Hypertension According to the Change in Intraocular Pressure (IOP) After Low-Intensity Laser IrRadiation (LILI) in Subjects with a Baseline IOP $\leq 21$ mm Hg

\begin{tabular}{|c|c|c|c|c|c|c|c|c|c|}
\hline \multicolumn{2}{|c|}{$\mathrm{IOP}(\mathrm{mm} \mathrm{Hg})$} & \multicolumn{2}{|c|}{ Change in IOP } & \multirow[b]{2}{*}{ Grade (risk) } & \multicolumn{2}{|c|}{$\mathrm{IOP}(\mathrm{mm} \mathrm{Hg})$} & \multicolumn{2}{|c|}{ Change in IOP } & \multirow[b]{2}{*}{ Grade (risk) } \\
\hline Baseline & $\begin{array}{c}\text { After } \\
\text { LILI }\end{array}$ & $\begin{array}{c}m m \\
\mathrm{Hg}\end{array}$ & $\%$ & & Baseline & $\begin{array}{l}\text { After } \\
\text { LILI }\end{array}$ & $\begin{array}{c}\mathrm{mm} \\
\mathrm{Hg}\end{array}$ & $\%$ & \\
\hline \multirow[t]{13}{*}{21} & 20 & 1 & 4.5 & \multirow[t]{4}{*}{0 (normal) } & \multirow[t]{9}{*}{17} & 16 & 1 & 5.9 & \multirow[t]{3}{*}{0 (normal) } \\
\hline & 19 & 2 & 9.5 & & & 15 & 2 & 11.8 & \\
\hline & 18 & 3 & 14.3 & & & 14 & 3 & 17.6 & \\
\hline & 17 & 4 & 19.0 & & & 13 & 4 & 23.5 & \multirow{2}{*}{ I (mild) } \\
\hline & 16 & 5 & 23.1 & \multirow{2}{*}{ I (mild) } & & 12 & 5 & 29.4 & \\
\hline & 15 & 6 & 28.6 & & & 11 & 6 & 35.3 & II (moderate) \\
\hline & 14 & 7 & 33.3 & \multirow[t]{2}{*}{ II (moderate) } & & 10 & 7 & 41.2 & \multirow[t]{3}{*}{ III (severe) } \\
\hline & 13 & 8 & 38.1 & & & 9 & 8 & 47.1 & \\
\hline & 12 & 9 & 42.8 & \multirow[t]{5}{*}{ III (severe) } & & 8 & 9 & 52.9 & \\
\hline & 11 & 10 & 47.6 & & \multirow[t]{4}{*}{16} & 15 & 1 & 6.3 & \multirow[t]{3}{*}{0 (normal) } \\
\hline & 10 & 11 & 52.4 & & & 14 & 2 & 12.5 & \\
\hline & 9 & 12 & 57.1 & & & 13 & 3 & 18.8 & \\
\hline & 8 & 13 & 61.9 & & & 12 & 4 & 25.0 & I (mild) \\
\hline \multirow[t]{12}{*}{20} & 19 & 1 & 5.0 & \multirow[t]{3}{*}{0 (normal) } & & 11 & 5 & 31.3 & \multirow[t]{2}{*}{ II (moderate) } \\
\hline & 18 & 2 & 10.0 & & & 10 & 6 & 37.5 & \\
\hline & 17 & 3 & 15.0 & & & 9 & 7 & 43.8 & III (severe) \\
\hline & 16 & 4 & 20.0 & I (mild) & & 8 & 8 & 50.0 & \\
\hline & 15 & 5 & 25.0 & & 15 & 14 & 1 & 6.7 & 0 (normal) \\
\hline & 14 & 6 & 30.0 & II (moderate) & & 13 & 2 & 13.3 & \\
\hline & 13 & 7 & 35.0 & & & 12 & 3 & 20.0 & I (mild) \\
\hline & 12 & 8 & 40.0 & III (severe) & & 11 & 4 & 26.7 & \\
\hline & 11 & 9 & 45.0 & & & 10 & 5 & 33.3 & II (moderate) \\
\hline & 10 & 10 & 50.0 & & & 9 & 6 & 40.0 & III (severe) \\
\hline & 9 & 11 & 55.0 & & & 8 & 7 & 46.7 & \\
\hline & 8 & 12 & 60.0 & & 14 & 13 & 1 & 7.1 & 0 (normal) \\
\hline 19 & 18 & 1 & 5.3 & 0 (normal) & & 12 & 2 & 14.3 & \\
\hline & 17 & 2 & 10.5 & & & 11 & 3 & 21.4 & I (mild) \\
\hline & 16 & 3 & 15.8 & & & 10 & 4 & 28.6 & \\
\hline & 15 & 4 & 21.1 & I (mild) & & 9 & 5 & 35.7 & II (moderate) \\
\hline & 14 & 5 & 26.3 & & & 8 & 6 & 42.9 & III (severe) \\
\hline & 13 & 6 & 31.6 & II (moderate) & 13 & 12 & 1 & 7.7 & 0 (normal) \\
\hline & 12 & 7 & 36.8 & & & 11 & 2 & 15.4 & \\
\hline & 11 & 8 & 42.1 & III (severe) & & 10 & 3 & 23.1 & I (mild) \\
\hline & 10 & 9 & 47.4 & & & 9 & 4 & 30.8 & II (moderate) \\
\hline & 9 & 10 & 52.6 & & & 8 & 5 & 38.5 & \\
\hline & 8 & 11 & 57.9 & & 12 & 11 & 1 & 8.3 & 0 (normal) \\
\hline 18 & 17 & 1 & 5.6 & 0 (normal) & & 10 & 2 & 16.7 & \\
\hline & 16 & 2 & 11.1 & & & 9 & 3 & 25.0 & I (mild) \\
\hline & 15 & 3 & 16.7 & & & 8 & 4 & 33.3 & II (moderate) \\
\hline & 14 & 4 & 22.2 & I (mild) & 11 & 10 & 1 & 9.1 & 0 (normal) \\
\hline & 13 & 5 & 27.8 & & & 9 & 2 & 18.2 & \\
\hline & 12 & 6 & 33.3 & II (moderate) & & 8 & 3 & 27.3 & I (mild) \\
\hline & 11 & 7 & 38.9 & & 10 & 9 & 1 & 10.0 & 0 (normal) \\
\hline & 10 & 8 & 44.4 & III (severe) & & 8 & 2 & 20.0 & \\
\hline & 9 & 9 & 50.0 & & & & & & \\
\hline & 8 & 10 & 55.6 & & & & & & \\
\hline
\end{tabular}

$\mathrm{Hg}(-21.8 \% ; p<0.001)$ resulting in identical IOP levels in both eyes (group 2C). The remaining 37 eyes also exhibited a decrease of the mean IOP from 17.2 to $14.3 \mathrm{~mm} \mathrm{Hg}(-16.9 \%$; $p<0.001)$ but the initial IOP difference between left and right eye remained: the mean IOP was 13.0 and $15.4 \mathrm{~mm} \mathrm{Hg}$ for right and left eyes, respectively (group 2D).

\section{LILI effects were limited to the irradiated eye only}

In a separate group of 35 subjects only one randomly selected eye was irradiated to examine whether any changes occurred also in the nonirradiated eye. The IOP of the non- irradiated, contralateral eye did not change (data not shown) confirming that the effects of LILI were limited to the irradiated eye only. In addition, the mean IOP did not change $30 \mathrm{~min}$ after mock treatment in the control group (14.2 \pm $0.5 \mathrm{~mm} \mathrm{Hg}$ vs. $14.2 \pm 0.3 \mathrm{~mm} \mathrm{Hg}$ at baseline).

\section{Safety and tolerability}

The treatment with LILI was overall well accepted by the subjects; the light was still perceived at the selected wavelength but did not blind. No adverse effects were observed. 
Table 3. Classification of 167 Eyes with a Baseline IOP $\leq 21 \mathrm{MM}$ Hg (GROUP 2, SEE TABLE 1) ACCORDING TO THE RESUlts OF THE LOW-INTENSITY LASER IRRADIATION (LILI) TEST

\begin{tabular}{lcccc}
\hline & \multicolumn{4}{c}{ IOP decrease (\%) } \\
\cline { 2 - 5 } & $<20$ & $20-29$ & $30-39$ & $\geq 40$ \\
\hline Grade & 0 & I & II & III \\
No. of eyes (\%) & $116(69.5)$ & $25(15.0)$ & $21(12.5)$ & $5(3.0)$ \\
\hline
\end{tabular}

\section{Discussion}

The biological effects of LILI have been studied in a variety of settings but only a few experimental studies have addressed ophthalmologic diseases before. ${ }^{18}$ We report here for the first time that LILI of the limbus area of the eye may lower the IOP. The laser power used here does not cause thermal damage and may well reach the ciliary body and the trabecular meshwork as previously shown. ${ }^{18}$ The molecular mechanisms translating laser irradiation into biological effects are largely unknown but it is conceivable the laser photons may cause cell membrane hyperpolarization promoting the contractility of trabecular meshwork and ciliary muscle cells. $^{19}$

The IOP is the result of a complex balance of the production and outflow of the aqueous humor in the eye. There is considerable inter-individual variation of the physiological level of the IOP, and this level cannot be readily determined. Even a "statistically normotensive" IOP within the range of 10 to $21 \mathrm{~mm} \mathrm{Hg}$ may cause glaucomatous damage to particularly susceptible eyes with much lower physiological levels of IOP. This is consistent with the occurrence of normal tension glaucoma.

The major result of this study is the finding that the IOP can be lowered by LILI, presumably to the individual physiological level. To verify that the physiological IOP has been identified, it may be necessary to repeat LILI until the IOP cannot be reduced further. A study limitation is the lack of long-term observations after LILI. It is currently unknown, how long the effects of a single LILI treatment last. There are several potential implications: 1) latent ocular hypertension defined as a nonphysiological IOP within the "normotensive" range, can be detected before it may lead to glaucomatous damage, 2) an individual target IOP can be defined for antiglaucoma therapy, and 3) LILI may even be useful to distinguish glaucomatous from nonglaucomatous opticus atrophy (remains to be shown).

\section{LILI decreased IOP}

The largest decrease in IOP $(-26 \%)$ was observed in eyes with a pathological baseline IOP of $>21 \mathrm{~mm} \mathrm{Hg}$ which exhibited ocular hypertension without damage. However, LILI also decreased IOP in most eyes $(73 \%)$ with a "normotensive" baseline IOP reaching a mean level of $14.1 \mathrm{~mm} \mathrm{Hg}$ in this group, which is in agreement with the accepted population-based normative IOP. ${ }^{16}$ Different patterns of IOP reduction suggested that 1) the physiological IOP may be lower in many, yet apparently normal eyes and that 2) these physiological IOP levels may differ between left and right eye or, conversely, the IOP may have been elevated in one eye only at baseline examination. Moreover, the effects of LILI were limited to the treated eye only, consistent with the independent blood supply and innervation of the eyes. Systemic effects of LILI were therefore not detectable.

\section{LILI may be useful to classify ocular hypertension}

The relative (not absolute) reduction of IOP compared to baseline values may be exploited for diagnostic purposes. We hypothesized, for example, that a baseline IOP of $12 \mathrm{~mm}$ $\mathrm{Hg}$ would be a potential risk factor for glaucoma if the IOP was $8 \mathrm{~mm} \mathrm{Hg}$ after LILI. In this case, the baseline value was increased by $33.3 \%$. In comparison, a decrease from 21 to $17 \mathrm{~mm} \mathrm{Hg}$ (i.e., $4 \mathrm{~mm} \mathrm{Hg}$ or $19.0 \%$ ) may raise no concern. This hypothesis was based on the finding that some eyes are particularly susceptible to glaucomatous damage, although they exhibit only low, "statistically normotensive" IOP levels within the range of 10 to $21 \mathrm{~mm} \mathrm{Hg}$. The prevalence of normal tension glaucoma is $2 \% .^{20-22}$

The diagnostic "LILI test" introduced here, may be particularly useful to detect latent ocular hypertension in eyes with apparently "normotensive" but potentially nonphysiological IOP, if ocular hypertension is associated with an open angle. Eyes with ocular hypertension may be at risk of developing glaucoma later on. Untreated ocular hypertension leads to glaucoma in $9.5 \%$ of cases. ${ }^{6,20,23}$ Eyes with ocular hypertension should be followed closely and treated early as soon as first signs of glaucomatous damage appear, with a therapeutic target IOP at the level observed after LILI.

Based on the relative reduction of baseline IOP after LILI, we propose a classification of ocular hypertension to be validated in future prospective clinical trials: a relative difference $<20 \%$ between IOPs before and after LILI would be considered normal variation (grade 0). Differences of 20-29\% (grade I) and 30-39\% (grade II) would correspond to mild and moderate ocular hypertension, respectively. A marked difference equal or greater than $40 \%$ would designate severe ocular hypertension (grade III). This classification could also be applied to eyes with latent ocular hypertension (baseline IOP ranging from 10 to $21 \mathrm{~mm} \mathrm{Hg}$ ) as shown in Table 2 .

According to this classification, no ocular hypertension was found in 116 of 167 eyes $(69.5 \%)$ of group 2 (Table 3 ). The remaining 51 eyes $(30.5 \%)$ are classified as eyes with mild (grade I), moderate (grade II), or severe (grade III) latent ocular hypertension relative to their lower physiological IOP. Notably, according to current terminology these eyes were normotensive without any further differentiation.

Latent ocular hypertension was found in $30.5 \%$ of eyes with "normotensive" IOP (Table 3). This may be rather frequent in comparison to published epidemiological data. It cannot be ruled out that the upper threshold for normal variation $(<20 \%$ change in IOP $)$ is too low. It should be noted that this classification is based on the limited data set from the present study. The classification may contain minor errors because baseline IOP may vary. However, the prevalence of glaucoma with permanent damage is estimated to range from $1.2 \%$ to $5.6 \%$; in more than half of these patients, their eyes exhibited a still "normotensive" IOP of $\leq 21 \mathrm{~mm} \mathrm{Hg}^{22}$ The frequency of severe latent ocular hypertension (grade III) in group 2 (Table 3 ) is in agreement with this observation. 


\section{Conclusion and Summary}

Our data suggested that LILI can reduce IOP in hypertensive and normotensive eyes. This effect may be exploited for a diagnostic test useful for the detection of ocular hypertension assuming that LILI uncovers the true physiological IOP. The LILI test may also provide valuable information about the individual therapeutic target IOP for treatment of ocular hypertension and glaucoma. Moreover, LILI itself may be an additional tool to treat ocular hypertension and glaucoma. On the basis of this pilot study, multi-center clinical trials are now required to confirm these data.

\section{Disclosure Statement}

No competing financial interests exist.

\section{References}

1. Foster, P.J., Buhrmann, R., Quigley, H.A., and Johnson, G.J. (2002). The definition and classification of glaucoma in prevalence surveys. Br. J. Ophthalmol. 86, 238-242.

2. Kahn, H.A., and Milton, R.C. (1980). Alternative definition of open-angle glaucoma: effect on prevalence and association in the Framingham Eye Study. Arch. Ophthalmol. 98, 2172-2177.

3. Advanced Glaucoma Intervention Study (AGIS). (2000). The relationship between control of interlobular pressure and visual field deterioration. Am. J. Ophthalmol. 130, 429440.

4. Anderson, D.R. (1989). Glaucoma. The damage caused by pressure. XLVI Edward Jackson memorial lecture. Am. J. Ophthalmol. 108, 485-495.

5. Heijl, A., Leske, C., Bergtsson, B., Hyman, L., Bengtsson, B., and Hussein, M. (2002). Early manifest glaucoma trial group. Reduction of intraocular pressure and glaucoma progression. Arch. Opthalmol. 120, 1268-1279.

6. Naskar, R., Wissing, M., and Thanos, S. (2002). Detection of early neuron degeneration and accompanying microglial responses in the retina of a rat model of glaucoma. Invest. Ophthalmol. Vis. Sci. 4, 2962-2968.

7. Armaly, M.F., Krueger, D.E., Maunder, L., et al. (1980). Biostatistical analysis of the collaborative glaucoma study. I. Summary report of the risk factors for glaucomatous visualfield defects. Arch. Ophthalmol. 98, 2163-2171.

8. Ocular Hypertension Treatment Study Group. (2002). The ocular hypertension treatment study. Arch. Ophthalmol. 120, 701-713.

9. Sommer, A., Tielsch, J.M., Katz, J., et al. (1991). Baltimore Eye Survey Research Group. Relationship between intraocular pressure and primary open angle glaucoma among white and black. Arch. Ophthalmol. 109, 1090-1095.

10. Lindenberg, T., Horn, F.K., and Korth, M. (2003). Multifokales Steady-state-Musterwechsel-ERG bei GlaukomPatienten. Ophthalmologe 100, 453-458.
11. Goldberg, I., Graham, S.L., and Klistorner, A.I. (2002) Multifocal objective perimetry in the detection of glaucomatous field loss. Am. J. Ophthalmol. 133, 29-39.

12. Jonson, C.A., and Samuels, S.J. (1997). Screening for glaucomatous visual field loss with frequency-doubling perimetry. Invest. Ophthalmol. Vis. Sci. 38, 413-425.

13. Paczka, J.A., Friedman, D.S., Quigley, H.A., Barron, Y., and Vitale, S. (2001). Diagnostic capabilities of frequencydoubling technology scanning laser polarimetry and nerve fiber layer photographs to distinguish glaucomatous damage. Am. J. Ophthalmol. 131, 188-197.

14. Asrani, S., Zeimer, R., Wilensky, J., Gieser, D., Vitale, S., and Lindenmuth, K. (2000). Large diurnal fluctuations in intraocular pressure are an independent risk factor in patients with glaucoma. Glaucoma 6, 134-142.

15. Demailly, P. (1989). Traitement actuel du glaucome primitif à angle ouvert. Masson, Paris.

16. Duke-Elder. (1989). System of Ophthalmology. The Physiology of the Eye and of Vision, Vol. 4, London: Henry Kimpton, pp. 239.

17. Karu T.I. (1987). Photobiological fundamentals of low-power laser therapy. IEEE J. Quantum Electronics 10, 1703-1717.

18. Ivandic, B.T., and Ivandic, T. (2008). Low-level laser therapy improves vision in patients with age-related macular degeneration. Photomed. Laser Surg. 26, 241-245.

19. Wiederholt, M., Schafer, R., Wagner, U., and LeppleWienhues A. (1996). Contractile response of the isolated trabecular meshwork and ciliary muscle to cholineric and adrenergic agents. Ger. J. Ophthalmol. 5, 146-153.

20. Collaborative normal-tension glaucoma study group. (1998). Comparison of glaucomatous progression between untreated patients with normal-tension glaucoma and patients with therapeutically reduced intraocular pressure. Am. J. Ophthalmol.126, 487-497.

21. Gordon, M.O., and Kass, M.A. (1999). Ocular hypertension treatment study group. Ocular hypertension treatment study. Arch. Ophthalmol. 117, 573-583.

22. Leske, C., Conell, A.M., Wu, S.Y., Hyman, L., and Schachat, A.P. (1997). Distribution of intraocular pressure. The Barbados eye study. Arch. Ophthalmol. 115, 1051-1057.

23. Thanos, S., and Naskar, R. (2004). Correlation between retinal ganglion cell death and chronically developing inherited glaucoma in a new rat mutant. Exp. Eye Res. 79, 119-129.

Address correspondence to: Boris T. Ivandic, M.D.

University of Heidelberg Otto-Meyerhof Centre Im Neuenheimer Feld 350 69120 Heidelberg Germany

E-mail: boris.ivandic@med.uni-heidelberg.de 
